\title{
Discovery of a meteoritic ejecta layer containing unmelted impactor fragments at the base of Paleocene lavas, Isle of
}

\section{Skye, Scotland}

Simon M. Drake ${ }^{1 *}$, Andrew D. Beard ${ }^{1}$, Adrian P. Jones ${ }^{2}$, David J. Brown ${ }^{3}$, A. Dominic Fortes ${ }^{4}$, lan L. Millar ${ }^{5}$, Andrew Carter $^{1}$, Jergus Baca $^{1}$ H. Downes ${ }^{1}$

${ }^{1}$ School of Earth and Planetary Sciences, Birkbeck College, University of London, Malet St, London, WC1E 7HX, UK. (*ubseaii@mail.bbk.ac.uk)

${ }^{2}$ Department of Earth Sciences, University College London, Gower St, London, WC1E 6BT, UK.

${ }^{3}$ School of Geographical and Earth Sciences, University of Glasgow, Lilybank Gardens, Glasgow, G12 $8 Q Q, U K$.

${ }^{4}$ ISIS Neutron Facility, Rutherford Appleton Laboratory, Harwell Science and Innovation Campus, Chilton, Didcot, Oxfordshire, OX11 OQX, UK.

${ }^{5}$ British Geological Survey, Natural Environment Research Council, Keyworth, Nottingham, NG12 $5 G C, U K$.

*Corresponding author: Simon. M. Drake (ubseaii@mail.bbk.ac.uk) 


\section{Supplementary Data: Methods}

\section{Zircon Sample preparation and U/Pb Zircon dating (Birkbeck College/University College London, UK)}

Zircons for $(\mathrm{U} / \mathrm{Th}) \mathrm{He}$ and $\mathrm{U} / \mathrm{Pb}$ dating from both sample sites were isolated using conventional mineral separation techniques. Native metal phases from both sites were then separated from crushed and sieved $(<300 \mu \mathrm{m})$ sub-samples using a strong neodymium (RE) magnet, sealed in plastic film to avoid contamination and to allow easier removal of extracted magnetic grains. Separated metal phases were then prepared as polished grain mounts for analysis on the Electron Microprobe (EMP) for chemical identification.

$\mathrm{U} / \mathrm{Pb}$ zircon ages from the An Carnach site were obtained using a New Wave $193 \mathrm{~nm}$ apertureimaged, frequency-quintupled laser ablation system coupled to an Agilent 7700 quadrupole-based ICP-MS. A typical laser operating condition for zircon uses an energy density of ca $2.5 \mathrm{~J} / \mathrm{cm}^{2}$ and a repetition rate of $10 \mathrm{~Hz}$. Repeated measurements of external zircon standard PLESOVIC (TIMS reference age 337.13 $\pm 0.37 \mathrm{Ma}$; (Sláma, et al., 2008) and NIST 612 silicate glass (Jochum et al., 2011) are used to correct for instrumental mass bias and depth-dependent inter-element fractionation of $\mathrm{Pb}$, Th and U. Temora (Black et al., 2003) and 91500 (Wiedenbeck et al., 2004) zircon were used as secondary age standards. Data are filtered using standard discordance tests with a $15 \%$ cut off. The ${ }^{206} \mathrm{~Pb} /{ }^{238} \mathrm{U}$ ratio is used to determine ages where $<1100 \mathrm{Ma}$ and the ${ }^{207} \mathrm{~Pb} /{ }^{206} \mathrm{~Pb}$ ratio for older grains. Data are processed using GLITTER 4.4 data reduction software. Time-resolved signals that record evolving isotopic ratios with depth in each crystal enabled filtering to remove spurious signals owing to overgrowth boundaries, inclusions, or fractures. 


\section{U/Pb Zircon dating for site 2 (NIGL facility, British Geological Survey, Keyworth, Nottingham, UK)}

$\mathrm{U} / \mathrm{Pb}$ zircon ages from the Torrin Road site were obtained using a New Wave Research UP193FX laser ablation system coupled to a Nu Instruments AttoM single-collector inductively coupled plasma mass spectrometer. Ablation parameters were optimized to suit the $\mathrm{Pb}$ and $\mathrm{U}$ contents of the material, and in all cases, bracketing reference materials were analysed using the same parameters; these were $5 \mathrm{~Hz}$, with a fluence of 1.5 to $3.0 \mathrm{~J} / \mathrm{cm} 2$, a 30 second ablation time, and a 25 to $35 \mu \mathrm{m}$ spot size. On the Attom, tuning was adopted that gave ThO and $\mathrm{UO}$ of $<0.4 \%$. Data processing for all analyses used the time-resolved function on the Nu Instruments' software, an in-house Excel spreadsheet for data reduction and error propagation, and Isoplot 4.15 for data presentation (Ludwig, 2003). Uncertainties were propagated in the manner advocated by Horstwood (2008). The Nu Attom SC-ICP-MS is used in peak-jumping mode with measurement on a MassCom secondary electron multiplier. The following masses are measured in each sweep: ${ }^{202} \mathrm{Hg},{ }^{204} \mathrm{~Pb}+\mathrm{Hg},{ }^{206} \mathrm{~Pb},{ }^{207} \mathrm{~Pb}$, $a^{2} d^{235} \mathrm{U}$. Each data integration records 100 sweeps of the measured masses, which roughly equates to 0.22 seconds. Dwell times on each mass are $400 \mu$ s on ${ }^{207} \mathrm{~Pb}$ and ${ }^{235} \mathrm{U}$, and $200 \mu$ s on all other masses; the switching between masses takes $40 \mu$ s. ${ }^{238} \mathrm{U}$ is calculated using ${ }^{238} \mathrm{U} /{ }^{235} \mathrm{U}=137.818$.

Three zircon reference materials (91500, GJ-1 and Plesovice) were analysed at regular intervals; the bias of the ${ }^{207} \mathrm{~Pb} /{ }^{206} \mathrm{~Pb}$ and ${ }^{206} \mathrm{~Pb} /{ }^{238} \mathrm{U}$ ratios of the 91500 standard from preferred values derived by TIMS analysis are used for normalization. ${ }^{206} \mathrm{~Pb} /{ }^{238} \mathrm{U}$ and ${ }^{207} \mathrm{~Pb} /{ }^{206} \mathrm{~Pb}$ uncertainties were propagated in a similar way utilising the measurement uncertainty and the reproducibility of the 91500 reference material. All zircons were CL-imaged prior to laser ablation work.

\section{Ar-Ar dating (Open University, Milton Keynes, UK)}

Plagioclase mineral separates were cleaned ultrasonically in acetone and then de-ionised water, dried using a hot plate and packaged in an aluminium foil packet prior to irradiation. Samples were irradiated in the $5 \mathrm{MW}$ medium flux reactor at McMaster Nuclear Reactor, McMaster University, Hamilton, Ontario and analysed in the Ar-Ar and Noble Gas laboratory at the Open University. Irradiation took place in the standard $5 \mathrm{C}$ position with $\mathrm{Cd}$ shielding and for $75 \mathrm{MWH}$. Neutron flux was 
monitored using biotite standard GA1550 and the $\mathrm{J}$ value was calculated $\mathrm{J}=0.00759 \pm 0.000038$, the error is included in the age calculations. Results were corrected for ${ }^{37} \mathrm{Ar}$ decay and neutron interference reactions. The following correction factors were used: $\left({ }^{39} \mathrm{Ar} /{ }^{37} \mathrm{Ar}\right)_{\mathrm{Ca}}=0.00065 \pm$ $0.00000325,\left({ }^{36} \mathrm{Ar}{ }^{37} \mathrm{Ar}\right)_{\mathrm{Ca}}=0.000265 \pm 0.00000133$, and $\left({ }^{40} \mathrm{Ar} /{ }^{39} \mathrm{Ar}\right)_{\mathrm{K}}=0.0085 \pm 0.0000425$, based on analyses of $\mathrm{K}$ and $\mathrm{C}$ salts.

The irradiated sample was mounted into an ultra-high vacuum system and step-heated using an SPI CW $1062 \mathrm{~nm}$ infra-red (IR) fibre laser. This laser was connected to a fully automated gas clean-up system, linked to a Mass Analyser Products (MAP) 215-50 noble gas spectrometer. Gas clean-up was achieved using two SAES AP10 getters, at room temperature and at $400{ }^{\circ} \mathrm{C}$, augmented by an in-line cold-trap cooled by liquid $\mathrm{N}_{2} .14$ heating steps were performed in total, and blank measurements were made either side of every two heating steps. The mass discrimination value used was 283 , calculated based on repeat measurements of air using an integrated calibration bottle. The age was calculated using Isoplot 3 (Ludwig, 2003) and reported at the $2 \sigma$ level (including the error on

$\mathrm{J}$ value). The data exceed the plateau criteria of at least $50 \%$ of the ${ }^{39} \mathrm{Ar}$ released in at least 4 consecutive steps $\left(80.6 \%\right.$ of ${ }^{39} \mathrm{Ar}$ released).

\section{Geochronology References}

Black, L. P., Kamo, S. L., Allen, C. M., Aleinikoff, J. N., David, D. W., Korsch, R. J. Foudoulis, C., 2003, Temora 1: a new zircon standard for Phanerozoic U-Pb geochronology: Chemical Geology, v. 200, p. 155-170, doi:10.1016/S0009-2541(03)00165-7.

Horstwood, M., 2008, Data reduction strategies, uncertainty assessment and resolution of LA-(MC-) ICP-MS isotope data, Mineralogical Association of Canada, v. 40, p. 283-303.

House, M.A., Farley, K.A., and Stockli, D, 2000, Helium chronometry of apatite and titanite using NdYAG laser heating. Earth and Planetary Science Letters, v. 183, p. 365-368.

Jochum, K. P., Weis, U., Stoll, B., Kuzmin, D., Yang, Q., Raczek, I., Jacob, D. E., Stracke, A., Birbaum, K., Frick, D. A., Günther, D. and Enzweiler, J., 2011, Determination of Reference Values for NIST SRM 610617 Glasses Following ISO Guidelines: Geostandards and Geoanalytical Research, v. 35, p. 397-425. 
Ludwig, K. R., 2003, Isoplot/EX version 3.0, A geochronological toolkit for Microsoft Excel: Berkeley Geochronology Center Special Publication.

Wiedenbeck, M., Hanchar, J. M., Peck, W. H., Sylvester, P., Valley, J., Whitehouse, M., Kronz, A., Morishita, Y., Nasdala, L., Fiebig, J., Franchi, I., Girard, J.-P., Greenwood, R.C., Hinton, R., Kita, N., Mason, P.R.D., Norman, M., Ogasawara, M., Piccoli, P.M., Rhede, D., Satoh, H., Schulz-Dobrick, B., Skår, O., Spicuzza, MJ., Terada, K., Tindle, A., Togashi, S., Vennemann, T., Xie, Q. and Zheng, Y.-F., 2004, Further Characterisation of the 91500 Zircon Crystal: Geostandards and Geoanalytical Research, v. 28, p. 9-39, doi:10.1111/j.1751908X.2004.tb01041.

\section{Raman microscopy (University College London, UK)}

Raman spectra of the reidite/zircon were acquired using a B\&WTek i-Raman plus portable spectrometer, equipped with a $532 \mathrm{~nm}$ laser ( $37 \mathrm{~mW}$ power at the fiber-optic probe tip) to stimulate Raman scattering, which is measured in the range $170-4000 \mathrm{~cm}^{-1}$ with spectral resolution of $3 \mathrm{~cm}^{-1}$.

Reidite was first discovered by high pressure experimentation (Reid and Ringwood, 1969), and was later formed by in-situ laser heating (Knittle and Williams, 1993) and shock experiments on zircon (Leroux et al., 1999). Reidite can be detected by Raman microscopy and infrared analysis (Gucsik et al., 2004) or by electron backscatter diffraction (EBSD), and focused ion beam imaging (Reddy et al., 2015; Erickson et al., 2017).

We note that slight shifts between 800 and 890 were recorded by Knittle and Williams (1993) as being due to changes in $\mathrm{Si}-\mathrm{O}$ bond strength because of an increase in bond length, a change in electron density function or a charge transfer between $\mathrm{O}$ and $\mathrm{Zr}$ or $\mathrm{O}$ and $\mathrm{Si}$. These workers used 'transparent' zircons during their high pressure shock experiments. It is unknown whether inclusions in natural zircons would influence band shifts in the 800-900 range when instantaneously shocked. Only five recorded terrestrial sites of natural reidite (supplementary file Tab.DR5) have been documented, ranging from 50 ka to $1.18 \mathrm{Ga}$ in age (Reddy et al., 2015). 


\section{Reidite References}

Erickson, T. M., Pearce, M. A., Reddy, S. M., Timms, N. E., Cavosie, A. J., Bourdet, J., Rickard W. D. A., Nemchin, A. A., 2017, Microstructural constraints on the mechanisms of the transformation to reidite in naturally shocked zircon: Contributions to Mineralogy and Petrology, v. 172(6), doi:10.1007/s00410-016-1322-0.

Gucsik, A., Koeberl, C., Brandstätter, F., Libowitzky, E. and Reimold, W. U., 2004, Cathodoluminescence, electron microscopy and Raman spectroscopy of experimentally shock metamorphosed zircon crystals and naturally shocked zircon from the Ries impact crater: in Dypvik, H., et al., (eds.), Cratering in marine environments and on ice: Berlin-Heidelberg, Springer, p. 281-322.

Leroux, H., Reimold, W. U., Koeberl, C., Hornemann, U., and Doukhan, J-C., 1999, Experimental shock deformation in zircon: A transmission electron microscopic study: Earth and Planetary Science Letters, v. 169, p. 291-301, doi:10.1016 /S0012 -821X (99) 00082-5.

Reddy, S. M., Johnson, T. E., Fisher, S., Rickard, W. D. A. and Taylor, R. J. M., 2015, Precambrian reidite discovered in shocked zircon from Stac Fada impactite Scotland: Geology, v. 43, p. 899-902, doi:10.1130/G37066.1.

Reid, A. F. and Ringwood, A. E., 1969, Newly observed high pressure transformations in Mn3O4, CaAl2O4, and ZrSiO4: Earth and Planetary Science Letters, v. 6, p. 205-208. 


\section{Electron microprobe analysis (Birkbeck College, London, UK)}

Major elemental analyses of the native metal, sulphide and phosphide phases were obtained using a Jeol JXA8100 Superprobe (Wavelength System) and an Oxford Instruments AZtec system (Energy System) at Birkbeck College. Analysis was carried out using an accelerating voltage of $15 \mathrm{kV}$, current of $1 \times 10^{-8} \mathrm{~A}$, and a beam diameter of $1 \mu \mathrm{m}$. Analyses were calibrated against standards of Spec-pure metals, natural sulphides, oxides and silicates, with the data corrected using a ZAF program.

Figure DR1: Individual zircon ages recovered from Skye impactoclastites and dated using laser ablation U-Pb methods. Site 1 (blue, $\sim 80$ analyses) and site 2 (red, $\sim 87$ analyses). The zircons record mostly Precambrian crystallisation ages. Note the presence of Triassic aged zircons at site 1. Their presence precludes any association with the $1.18 \mathrm{Ga}$ Stac Fada event.

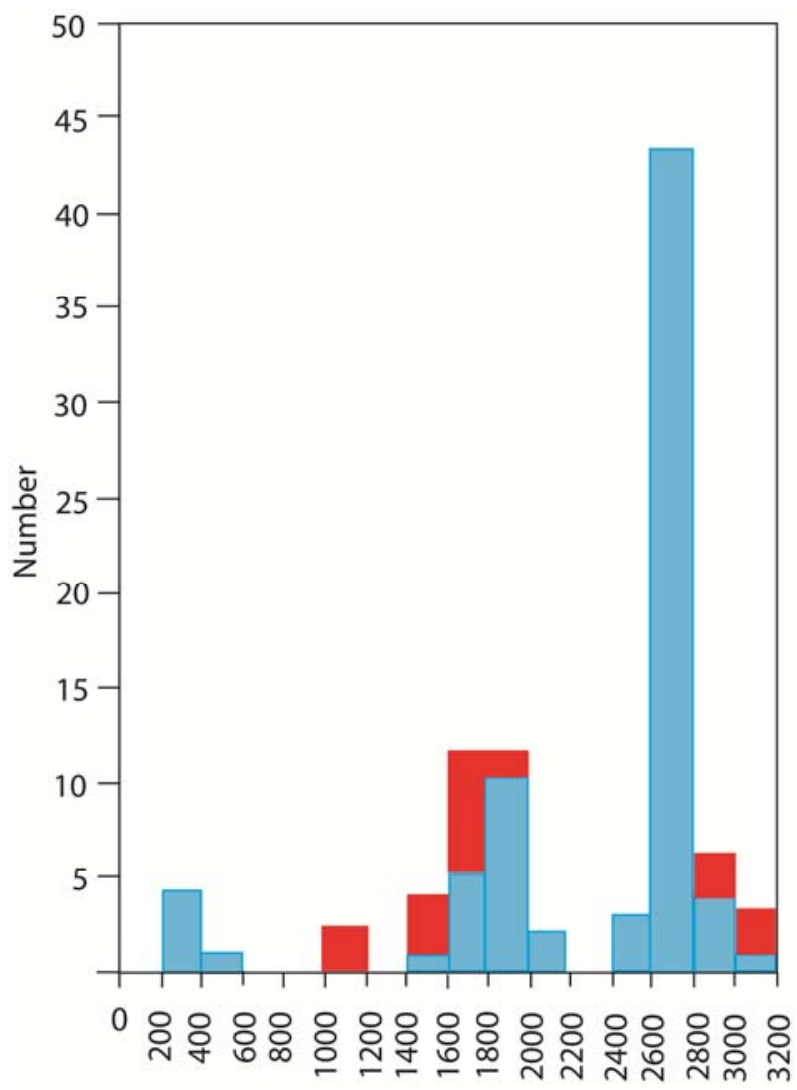

Age in millions of years 
Figure DR2: Vertical logged sections of Impactoclastite deposits at site 1 and site 2.

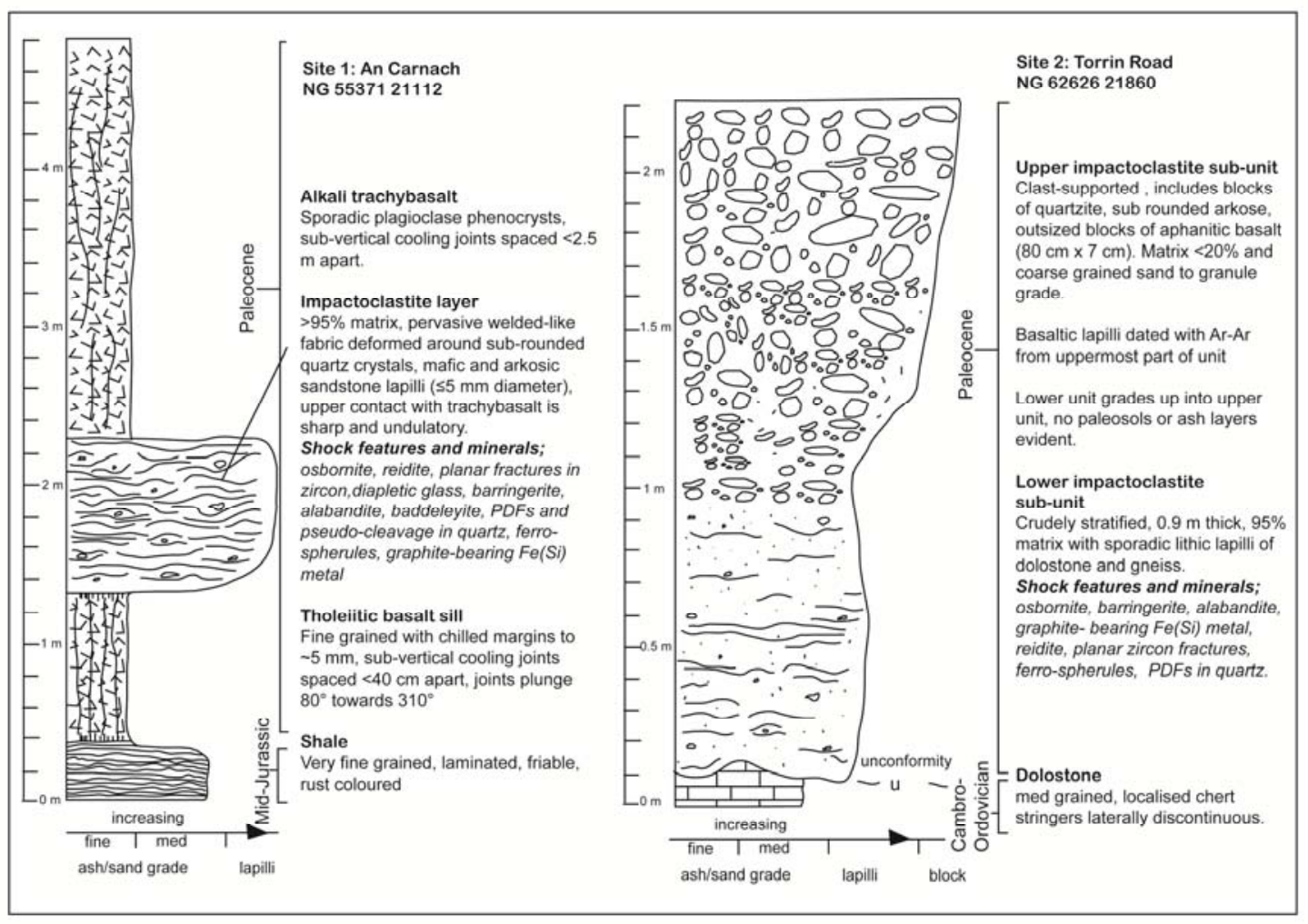


Figure DR3: Plateau age of basaltic clast incorporated in impactoclastite at site 2

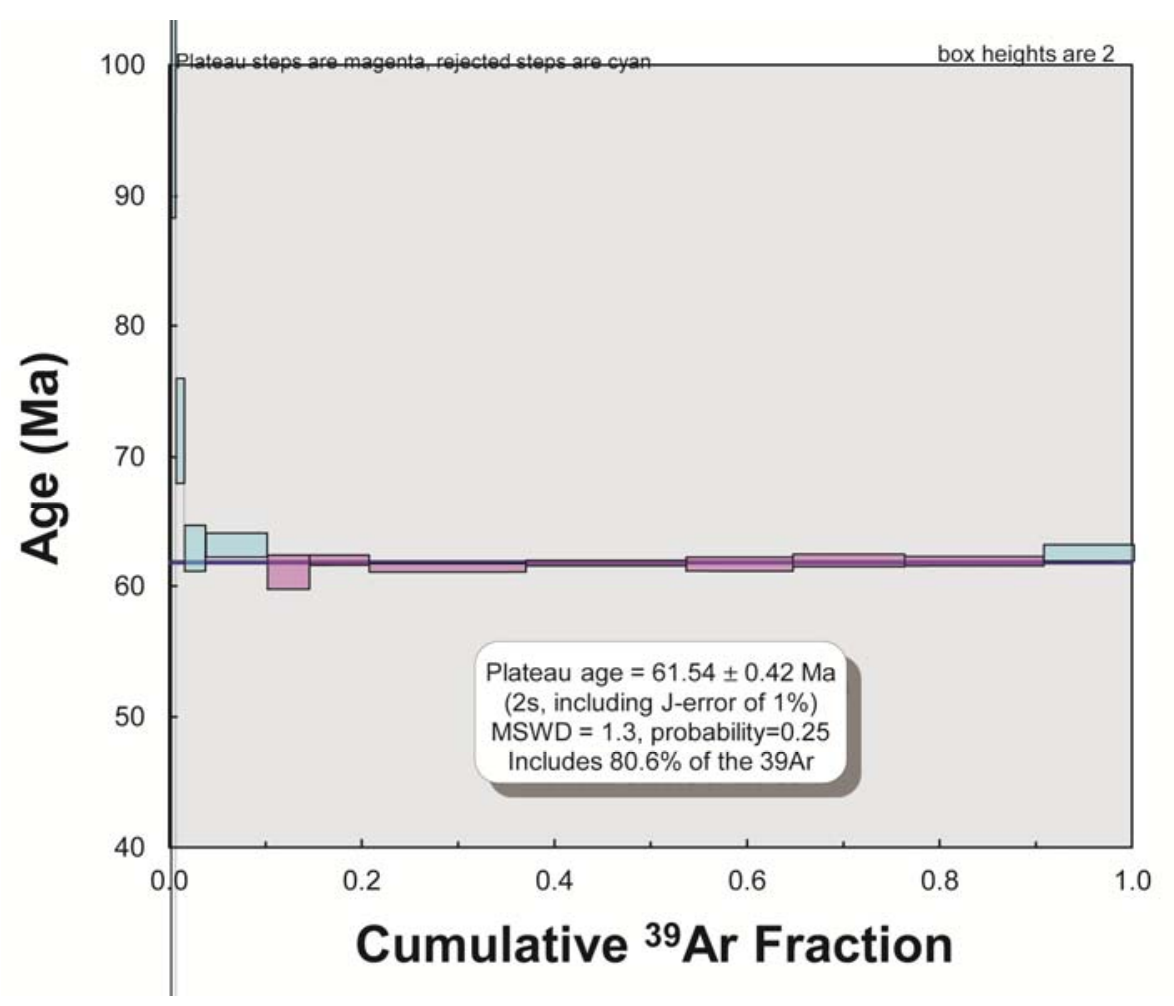


Figure DR4: Reidite peaks for site 1 and site 2 Isle of Skye together with peaks for natural zircon and synthetically derived reidite

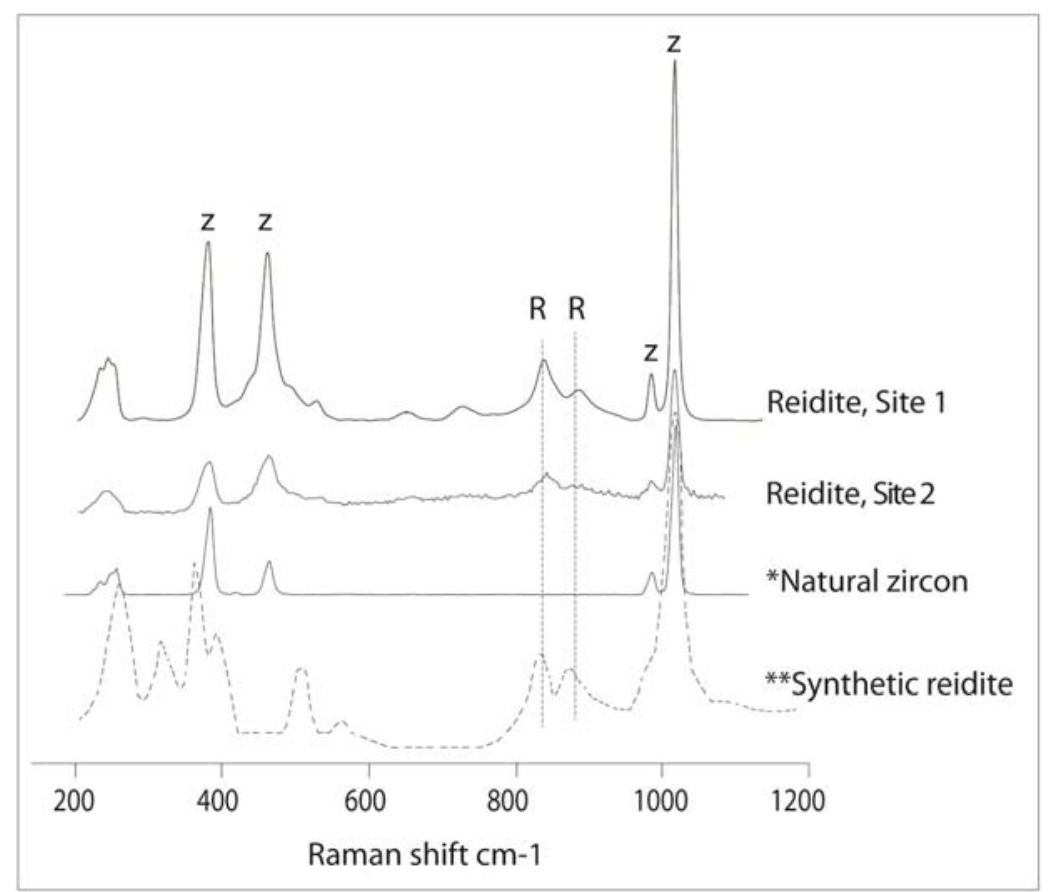

* Knittle, E. and Williams, Q., 1993 High-pressure Raman spectroscopy of $\mathrm{ZrSiO}_{4}$ : Observation of the zircon to scheelite transition at $300 \mathrm{~K}$ : Am Mineral v. 78, p. 245-252.

** Raman Spectrum http://rruff.info/Zircon/R050203 
Figure DR5: Concordia plots of zircon ages at site 2 lower unit near the Chambered Cairn

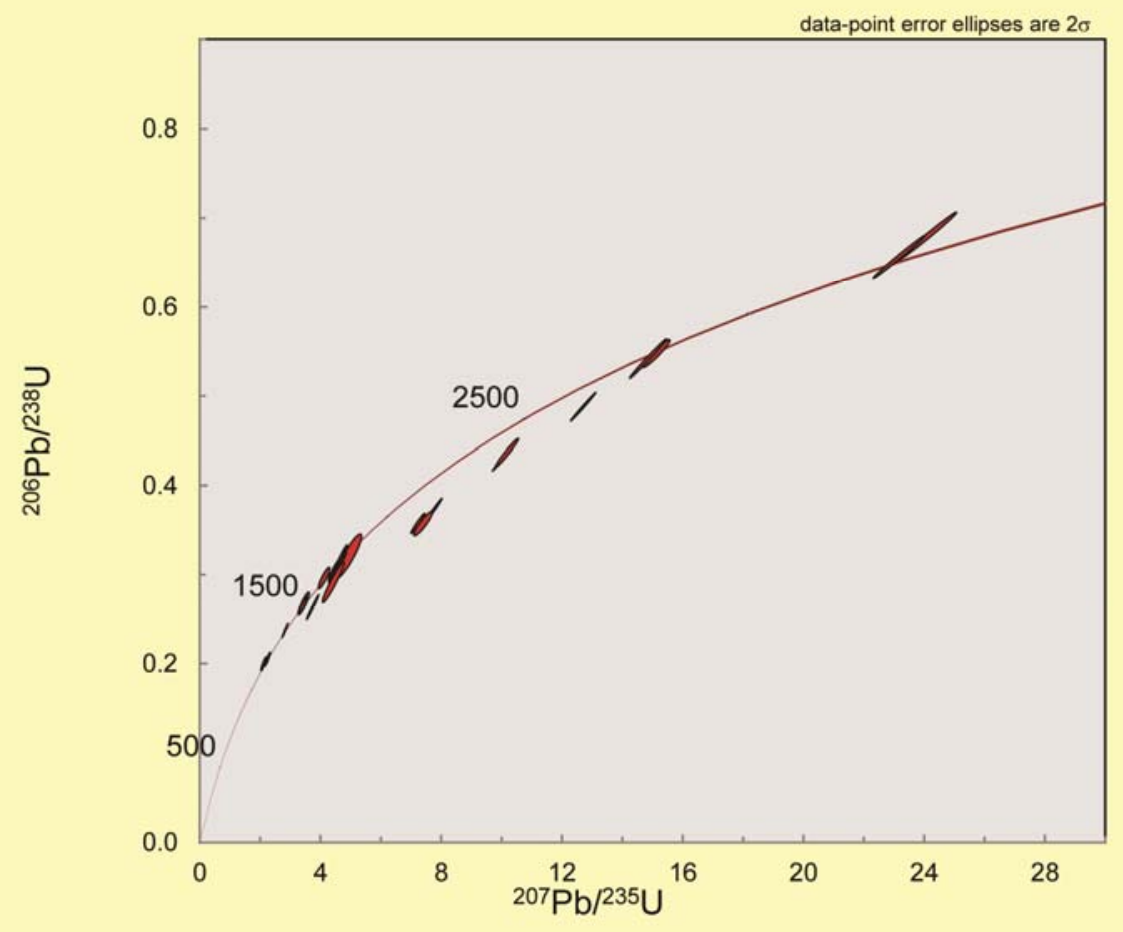



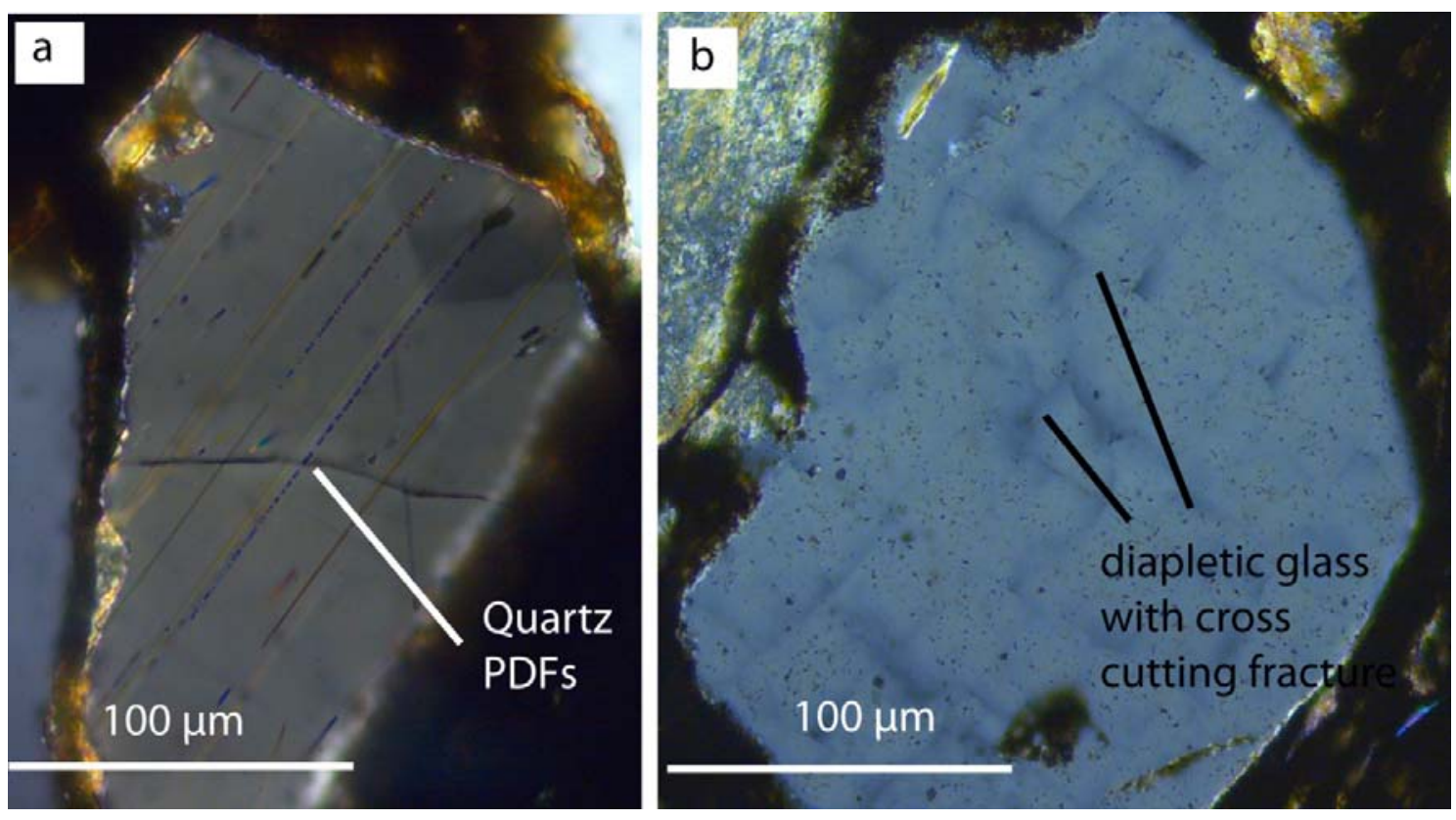

a) Quartz grain from site 1, with both fresh and decorated sets of Planar Deformation Features (PDFs). The decorated PDFs indicate some degree of re-crystallisation has occurred, with the original PDF now appearing as straight arrays of fluid inclusions.

b) Quartz grain from site 1 exhibiting Planar Fractures (PFs) forming a pseudo-cleavage with areas of diaplectic glass. Such glass commonly occurs at the PFs intersections and indicate derivation from instantaneous high pressure shock at 30-50 GPa ( ${ }^{\star}$ French and Koeberl, 2010).

* French, B.M., and Koeberl, C., 2010 The convincing identification of terrestrial meteorite impact structures: What works, what doesn't, and why: Earth Science Reviews, v. 98, p. 123-170, doi:10.10.16/j.earscirev.2009.10.009. 
Table DR1 : Step heating ages (highlighted in yellow) of basaltic clast at site 2 (near Chambered Cairn- upper unit). $\mathrm{J}=0.007608838$

\begin{tabular}{|c|c|c|c|c|c|c|c|c|c|c|c|}
\hline Run No & Comment & 40Ar & $+/-$ & $39 \mathrm{Ar}$ & $+/-$ & $38 A r$ & +- & $37 \mathrm{Ar}$ & +- & $36 \mathrm{Ar}$ & $+/-$ \\
\hline 12A62218 & Step 1 & 0.0077206 & 0.0007437 & 3.52E-05 & 3.45E-05 & 8.47E-06 & 1.39E-05 & 0.000462 & 0.0002823 & 2.13E-05 & $1.19 \mathrm{E}-05$ \\
\hline 12A62219 & Step 2 & 0.1174556 & 0.0007804 & 0.0008816 & 4.15E-05 & 6.98E-05 & 1.39E-05 & 0.0029385 & 0.0002824 & 0.0002506 & 1.19E-05 \\
\hline 12A62221 & Step 3 & 0.6037719 & 0.0010273 & 0.0162696 & 7.75E-05 & 0.0005093 & 2.25E-05 & 0.0351579 & 0.0002826 & 0.0013921 & 2.10E-05 \\
\hline 12A62222 & Step 4 & 0.826142 & 0.0008843 & 0.0416012 & 0.000117 & 0.0009488 & 2.25E-05 & 0.075171 & 0.0002827 & 0.0020415 & 2.10E-05 \\
\hline 12A62224 & Step 5 & 1.4962781 & 0.0014745 & 0.0944759 & 0.0002087 & 0.002114 & 3.21E-05 & 0.2689395 & 0.0002829 & 0.0035702 & 2.10E-05 \\
\hline 12A62226 & Step 6 & 2.8966043 & 0.0042113 & 0.2820416 & 0.0004146 & 0.0052926 & 5.20E-05 & 0.7303898 & 0.0002832 & 0.0053279 & 3.07E-05 \\
\hline 12A62229 & Step 7 & 1.4950603 & 0.0009783 & 0.1848611 & 0.0003527 & 0.0031667 & 4.19E-05 & 0.3652978 & 0.0002835 & 0.0022246 & 3.07E-05 \\
\hline 12A62232 & Step 8 & 1.6550157 & 0.0015328 & 0.2702872 & 0.0003831 & 0.0043544 & $5.14 \mathrm{E}-05$ & 0.6055781 & 0.0002643 & 0.0013912 & 1.07E-05 \\
\hline 12A62234 & Step 9 & 4.2513418 & 0.0033718 & 0.7072563 & 0.000576 & 0.0107833 & 6.16E-05 & 1.4445159 & 0.0002645 & 0.0034789 & 3.03E-05 \\
\hline $12 A 62237$ & Step 10 & 4.145167 & 0.0020956 & 0.7188705 & 0.0005555 & 0.0107833 & 9.21E-05 & 1.2374778 & 0.0002648 & 0.0028737 & 2.04E-05 \\
\hline 12A62238 & Step 11 & 2.8387972 & 0.0020086 & 0.4792595 & 0.0005148 & 0.0072878 & 4.12E-05 & 1.1179034 & 0.000265 & 0.0021854 & 3.03E-05 \\
\hline $12 A 62240$ & Step 12 & 2.9748736 & 0.0030579 & 0.5001037 & 0.0001796 & 0.0074718 & 4.12E-05 & 1.1411486 & 0.0002652 & 0.0022793 & 3.03E-05 \\
\hline 12A62241 & Step 13 & 3.9264884 & 0.0044144 & 0.6326591 & 0.0008837 & 0.0094444 & 8.19E-05 & 1.2431118 & 0.0002653 & 0.0034522 & 2.04E-05 \\
\hline $12 A 62243$ & Step 14 & 2.9077482 & 0.0022316 & 0.4050682 & 0.0006475 & 0.0064599 & 7.18E-05 & 1.0550581 & 0.0002656 & 0.0034721 & 3.03E-05 \\
\hline
\end{tabular}

\begin{tabular}{|c|c|c|c|c|c|c|c|c|c|c|c|c|c|c|}
\hline Run No & Comment & $40 A r^{\star} / 39 A r$ & $+/-$ & Age & $+/-$ & $\begin{array}{l}+/ \text {-(no J } \\
\text { error) }\end{array}$ & $39 / 40$ & $+/-$ & $36 / 40$ & $+/-$ & $37 / 39$ & $+/-$ & $38 / 39$ & $+1-$ \\
\hline 12A62218 & Step 1 & 40.522344 & 109.42472 & 484.77396 & 1147.9343 & 1147.9324 & 0.0045536 & 0.0044847 & 0.0027597 & 0.0015599 & 13.142534 & 15.178657 & 0.2408855 & 0.4595569 \\
\hline 12A62219 & Step 2 & 49.218082 & 4.6876865 & 573.76612 & 46.874215 & 46.809756 & 0.0075055 & 0.0003568 & 0.002134 & 0.000102 & 3.333262 & 0.3566748 & 0.079171 & 0.0161591 \\
\hline 12A62221 & Step 3 & 11.825921 & 0.3906128 & 155.41552 & 4.9745444 & 4.9185101 & 0.0269466 & 0.0001362 & 0.0023057 & 3.50E-05 & 2.1609585 & 0.020188 & 0.0313032 & 0.00139 \\
\hline 12A62222 & Step 4 & 5.3574522 & 0.1513975 & 72.070073 & 2.0275109 & 1.9965011 & 0.050356 & 0.0001516 & 0.0024711 & 2.56E-05 & 1.806945 & 0.0084871 & 0.0228067 & 0.0005443 \\
\hline 12A62224 & Step 5 & 4.6709877 & 0.0682921 & 62.994777 & 0.9565856 & 0.9051199 & 0.0631406 & 0.0001527 & 0.002386 & 1.42E-05 & 2.8466454 & 0.006964 & 0.0223757 & 0.0003429 \\
\hline $12 A 62226$ & Step 6 & 4.688022 & 0.036114 & 63.220529 & 0.5705535 & 0.4785827 & 0.0973697 & 0.0002013 & 0.0018394 & 1.09E-05 & 2.5896529 & 0.0039368 & 0.0187655 & 0.0001863 \\
\hline 12A62229 & Step 7 & 4.5314231 & 0.0500733 & 61.144087 & 0.7291774 & 0.6643347 & 0.1236479 & 0.0002494 & 0.001488 & 2.05E-05 & 1.9760667 & 0.0040702 & 0.0171302 & 0.0002292 \\
\hline 12A62232 & Step 8 & 4.6022133 & 0.0145472 & 62.083035 & 0.360996 & 0.192901 & 0.163314 & 0.0002765 & 0.0008406 & $6.51 \mathrm{E}-06$ & 2.2404984 & 0.003323 & 0.0161104 & 0.0001915 \\
\hline 12A62234 & Step 9 & 4.5575211 & 0.0140242 & 61.490303 & 0.3549279 & 0.186027 & 0.1663607 & 0.0001891 & 0.0008183 & 7.15E-06 & 2.042422 & 0.0017048 & 0.0152467 & 8.79E-05 \\
\hline 12A62237 & Step 10 & 4.5849404 & 0.0095625 & 61.853975 & 0.329418 & 0.1268188 & 0.1734238 & 0.0001601 & 0.0006933 & 4.94E-06 & 1.7214197 & 0.0013804 & 0.0150004 & 0.0001287 \\
\hline 12A62238 & Step 11 & 4.5758196 & 0.0197481 & 61.73301 & 0.4008476 & 0.2619171 & 0.1688248 & 0.0002171 & 0.0007698 & 1.07E-05 & 2.332564 & 0.0025656 & 0.0152064 & 8.76E-05 \\
\hline $12 \mathrm{~A} 62240$ & Step 12 & 4.601749 & 0.0189734 & 62.076877 & 0.3954609 & 0.2515951 & 0.1681092 & 0.000183 & 0.0007662 & 1.02E-05 & 2.281824 & 0.0009761 & 0.0149405 & $8.27 E-05$ \\
\hline $12 \mathrm{~A} 62241$ & Step 13 & 4.5938656 & 0.0134442 & 61.972338 & 0.3529409 & 0.1782862 & 0.1611259 & 0.0002889 & 0.0008792 & 5.29E-06 & 1.9648999 & 0.0027764 & 0.0149281 & 0.0001312 \\
\hline $12 \mathrm{A62243}$ & Step 14 & 4.6455136 & 0.0239353 & 62.657119 & 0.4421306 & 0.3172889 & 0.1393065 & 0.000247 & 0.0011941 & 1.04E-05 & 2.6046435 & 0.0042151 & 0.0159477 & 0.000179 \\
\hline
\end{tabular}


Table DR2: Zircon ages (highlighted in yellow) of site 1 (An Carnach) zircons

\begin{tabular}{|c|c|c|c|c|c|c|c|c|c|c|c|c|c|c|c|c|c|c|c|}
\hline \multirow[b]{2}{*}{$\begin{array}{l}\text { Grain } \\
\text { No. }\end{array}$} & \multirow[b]{2}{*}{$\begin{array}{c}\mathrm{Pb} \\
(\mathrm{ppm})\end{array}$} & \multirow[b]{2}{*}{$\begin{array}{c}U \\
(\mathrm{ppm})\end{array}$} & \multirow[b]{2}{*}{$\begin{array}{l}\text { Atomic } \\
\text { Th/U }\end{array}$} & \multicolumn{6}{|c|}{ Ratios } & \multicolumn{6}{|c|}{ Ages (Ma) } & \multirow{2}{*}{$\begin{array}{c}\% \\
\text { concord. } \\
(206 / 238 \\
207 / 235)\end{array}$} & \multirow{2}{*}{$\begin{array}{c}\% \\
\text { concord. } \\
(206 / 238 \\
207 / 206)\end{array}$} & \multirow[b]{2}{*}{$\begin{array}{l}\text { Best } \\
\text { Age }\end{array}$} & \multirow[b]{2}{*}{ $\pm 2 \mathrm{~s}$} \\
\hline & & & & 206/238 & \pm s.e. & $207 / 235$ & \pm s.e. & 207/206 & \pm s.e. & $206 / 238$ & $\pm 2 s$ & 207/235 & $\pm 2 s$ & 207/206 & $\pm 2 s$ & & & & \\
\hline G1 & 153.3 & 281.1 & 0.34 & 0.4989 & 0.0057 & 13.0620 & 0.1749 & 0.1900 & 0.0024 & 2609.0 & 49.1 & 2684.1 & 32.2 & 2741.7 & 23.9 & 2.8 & 4.8 & 2741.7 & 23.9 \\
\hline G2 & 56.1 & 102.1 & 0.45 & 0.4889 & 0.0058 & 12.6754 & 0.1891 & 0.1881 & 0.0027 & 2566.0 & 50.5 & 2655.8 & 35.2 & 2725.5 & 27.3 & 3.4 & 5.9 & 2725.5 & 27.3 \\
\hline G3 & 38.1 & 909.2 & 0.55 & 0.0389 & 0.0005 & 0.2879 & 0.0065 & 0.0537 & 0.0012 & 246.1 & 5.8 & 256.9 & 11.5 & 357.2 & 13.5 & 4.2 & 31.1 & 246.1 & 5.8 \\
\hline G4 & 152.5 & 236.4 & 0.29 & 0.5862 & 0.0068 & 18.9396 & 0.2588 & 0.2344 & 0.0030 & 2973.9 & 55.3 & 3038.7 & 33.5 & 3082.5 & 25.0 & 2.1 & 3.5 & 3082.5 & 25.0 \\
\hline G5 & 8.7 & 23.8 & 1.42 & 0.2705 & 0.0039 & 3.8046 & 0.1139 & 0.1021 & 0.0031 & 1543.2 & 40.0 & 1593.7 & 54.3 & 1661.9 & 49.8 & 3.2 & 7.1 & 1661.9 & 49.8 \\
\hline G6 & 14.3 & 42.0 & 0.23 & 0.3323 & 0.0043 & 5.3870 & 0.1112 & 0.1176 & 0.0024 & 1849.4 & 41.3 & 1882.8 & 41.7 & 1920.4 & 35.6 & 1.8 & 3.7 & 1920.4 & 35.6 \\
\hline G7 & 157.8 & 258.8 & 0.43 & 0.5328 & 0.0062 & 13.9589 & 0.1959 & 0.1901 & 0.0025 & 2753.1 & 52.2 & 2746.9 & 33.6 & 2743.0 & 25.3 & -0.2 & -0.4 & 2743.0 & 25.3 \\
\hline G8 & 96.4 & 384.6 & 0.32 & 0.2424 & 0.0028 & 3.0827 & 0.0449 & 0.0923 & 0.0013 & 1399.4 & 29.0 & 1428.4 & 27.6 & 1472.7 & 21.6 & 2.0 & 5.0 & 1472.7 & 21.6 \\
\hline G9 & 46.1 & 78.3 & 0.61 & 0.5009 & 0.0060 & 12.5955 & 0.1897 & 0.1825 & 0.0027 & 2617.5 & 51.4 & 2649.9 & 35.4 & 2675.4 & 27.4 & 1.2 & 2.2 & 2675.4 & 27.4 \\
\hline G10 & 89.8 & 146.9 & 0.85 & 0.4970 & 0.0060 & 12.8437 & 0.1990 & 0.1875 & 0.0028 & 2600.9 & 51.8 & 2668.3 & 36.3 & 2720.4 & 28.3 & 2.5 & 4.4 & 2720.4 & 28.3 \\
\hline G11 & 30.3 & 55.9 & 0.38 & 0.4925 & 0.0060 & 12.8181 & 0.2009 & 0.1888 & 0.0029 & 2581.6 & 51.7 & 2666.4 & 36.6 & 2731.9 & 28.8 & 3.2 & 5.5 & 2731.9 & 28.8 \\
\hline G12 & 39.9 & 68.5 & 0.45 & 0.5124 & 0.0061 & 13.3968 & 0.2037 & 0.1897 & 0.0028 & 2666.8 & 52.3 & 2708.0 & 35.8 & 2739.6 & 27.8 & 1.5 & 2.7 & 2739.6 & 27.8 \\
\hline G13 & 45.9 & 78.3 & 0.55 & 0.5060 & 0.0060 & 13.1689 & 0.1985 & 0.1888 & 0.0027 & 2639.5 & 51.7 & 2691.8 & 35.5 & 2732.1 & 27.5 & 1.9 & 3.4 & 2732.1 & 27.5 \\
\hline G14 & 89.5 & 155.3 & 0.49 & 0.5060 & 0.0059 & 13.2594 & 0.1868 & 0.1901 & 0.0025 & 2639.7 & 50.3 & 2698.3 & 33.4 & 2743.1 & 25.3 & 2.2 & 3.8 & 2743.1 & 25.3 \\
\hline G15 & 49.5 & 81.1 & 0.62 & 0.5132 & 0.0061 & 13.2343 & 0.1998 & 0.1871 & 0.0027 & 2670.2 & 52.1 & 2696.5 & 35.5 & 2717.0 & 27.5 & 1.0 & 1.7 & 2717.0 & 27.5 \\
\hline G16 & 133.5 & 229.4 & 0.55 & 0.5034 & 0.0059 & 12.9752 & 0.1852 & 0.1870 & 0.0025 & 2628.3 & 50.3 & 2677.9 & 33.8 & 2716.1 & 25.7 & 1.8 & 3.2 & 2716.1 & 25.7 \\
\hline G17 & 143.6 & 461.6 & 0.30 & 0.2986 & 0.0034 & 4.2819 & 0.0608 & 0.1040 & 0.0014 & 1684.5 & 34.1 & 1689.9 & 29.1 & 1697.4 & 22.2 & 0.3 & 0.8 & 1697.4 & 22.2 \\
\hline G18 & 72.6 & 118.9 & 0.62 & 0.5181 & 0.0061 & 13.8887 & 0.2014 & 0.1945 & 0.0027 & 2690.9 & 51.6 & 2742.1 & 34.4 & 2780.8 & 26.3 & 1.9 & 3.2 & 2780.8 & 26.3 \\
\hline G19 & 57.6 & 103.5 & 0.30 & 0.5071 & 0.0063 & 12.8199 & 0.2159 & 0.1835 & 0.0030 & 2644.0 & 54.1 & 2666.5 & 38.9 & 2684.3 & 31.1 & 0.8 & 1.5 & 2684.3 & 31.1 \\
\hline G2O & 111.9 & 362.3 & 0.10 & 0.3144 & 0.0037 & 5.0467 & 0.0800 & 0.1165 & 0.0018 & 1762.3 & 36.4 & 1827.2 & 32.7 & 1902.7 & 26.3 & 3.6 & 7.4 & 1902.7 & 26.3 \\
\hline G21 & 69.5 & 124.5 & 0.40 & 0.4993 & 0.0059 & 12.5234 & 0.1850 & 0.1820 & 0.0026 & 2610.6 & 50.6 & 2644.5 & 34.6 & 2671.2 & 26.6 & 1.3 & 2.3 & 2671.2 & 26.6 \\
\hline G22 & 68.9 & 109.1 & 0.62 & 0.5287 & 0.0064 & 13.7951 & 0.2130 & 0.1893 & 0.0028 & 2735.8 & 53.6 & 2735.7 & 36.2 & 2736.4 & 28.2 & 0.0 & 0.0 & 2736.4 & 28.2 \\
\hline G23 & 77.3 & 146.9 & 0.29 & 0.4899 & 0.0057 & 12.6140 & 0.1842 & 0.1868 & 0.0026 & 2570.2 & 49.7 & 2651.3 & 34.2 & 2714.5 & 26.3 & 3.1 & 5.3 & 2714.5 & 26.3 \\
\hline
\end{tabular}




\begin{tabular}{|c|c|c|c|c|c|c|c|c|c|c|c|c|c|c|c|c|c|c|c|}
\hline G24 & 10.6 & 208.4 & 0.72 & 0.0449 & 0.0006 & 0.3158 & 0.0117 & 0.0511 & 0.0019 & 282.8 & 7.5 & 278.7 & 19.4 & 245.3 & 16.3 & -1.5 & -15.3 & 282.8 & 7.5 \\
\hline G25 & 13.2 & 288.1 & 0.63 & 0.0412 & 0.0005 & 0.2927 & 0.0089 & 0.0515 & 0.0016 & 260.3 & 6.6 & 260.6 & 15.2 & 264.6 & 14.2 & 0.1 & 1.6 & 260.3 & 6.6 \\
\hline G26 & 9.0 & 191.6 & 0.82 & 0.0403 & 0.0006 & 0.2827 & 0.0119 & 0.0509 & 0.0022 & 254.8 & 7.2 & 252.8 & 20.1 & 235.9 & 17.9 & -0.8 & -8.0 & 254.8 & 7.2 \\
\hline G27 & 208.9 & 339.9 & 0.31 & 0.5560 & 0.0065 & 16.4409 & 0.2358 & 0.2146 & 0.0029 & 2849.9 & 53.6 & 2902.8 & 34.3 & 2940.5 & 26.1 & 1.8 & 3.1 & 2940.5 & 26.1 \\
\hline G28 & 167.9 & 327.3 & 0.43 & 0.4660 & 0.0054 & 11.6545 & 0.1682 & 0.1815 & 0.0025 & 2466.2 & 47.6 & 2577.1 & 33.5 & 2666.2 & 25.7 & 4.3 & 7.5 & 2666.2 & 25.7 \\
\hline G29 & 55.5 & 86.7 & 0.96 & 0.5057 & 0.0061 & 13.1276 & 0.2055 & 0.1884 & 0.0028 & 2638.2 & 52.0 & 2688.9 & 36.4 & 2727.9 & 28.5 & 1.9 & 3.3 & 2727.9 & 28.5 \\
\hline G30 & 97.7 & 159.5 & 0.66 & 0.5155 & 0.0060 & 13.6183 & 0.2020 & 0.1917 & 0.0027 & 2680.1 & 51.4 & 2723.5 & 34.7 & 2756.7 & 26.8 & 1.6 & 2.8 & 2756.7 & 26.8 \\
\hline G31 & 43.1 & 113.3 & 0.47 & 0.3468 & 0.0042 & 5.9547 & 0.1002 & 0.1246 & 0.0020 & 1919.4 & 39.9 & 1969.2 & 35.3 & 2022.7 & 28.7 & 2.5 & 5.1 & 2022.7 & 28.7 \\
\hline G32 & 104.2 & 184.6 & 0.28 & 0.5177 & 0.0061 & 13.7135 & 0.2031 & 0.1922 & 0.0027 & 2689.5 & 51.5 & 2730.1 & 34.7 & 2761.1 & 26.7 & 1.5 & 2.6 & 2761.1 & 26.7 \\
\hline G33 & 76.7 & 110.5 & 1.00 & 0.5331 & 0.0064 & 14.1278 & 0.2209 & 0.1923 & 0.0029 & 2754.5 & 53.7 & 2758.3 & 36.4 & 2761.8 & 28.5 & 0.1 & 0.3 & 2761.8 & 28.5 \\
\hline G34 & 84.5 & 134.3 & 0.41 & 0.5576 & 0.0066 & 16.6879 & 0.2510 & 0.2172 & 0.0031 & 2856.7 & 54.5 & 2917.1 & 35.6 & 2959.8 & 27.6 & 2.1 & 3.5 & 2959.8 & 27.6 \\
\hline G35 & 42.1 & 68.5 & 0.77 & 0.5043 & 0.0062 & 13.0330 & 0.2147 & 0.1875 & 0.0030 & 2632.1 & 52.7 & 2682.0 & 37.9 & 2720.7 & 30.2 & 1.9 & 3.3 & 2720.7 & 30.2 \\
\hline G36 & 74.1 & 162.2 & 0.23 & 0.4468 & 0.0053 & 11.7555 & 0.1828 & 0.1909 & 0.0029 & 2381.0 & 47.3 & 2585.1 & 35.7 & 2750.0 & 28.3 & 7.9 & 13.4 & 2750.0 & 28.3 \\
\hline G37 & 70.0 & 111.9 & 0.77 & 0.5140 & 0.0063 & 13.6441 & 0.2267 & 0.1926 & 0.0031 & 2673.7 & 53.6 & 2725.3 & 38.3 & 2764.6 & 30.5 & 1.9 & 3.3 & 2764.6 & 30.5 \\
\hline G38 & 111.1 & 205.6 & 0.36 & 0.4922 & 0.0058 & 12.7903 & 0.1932 & 0.1885 & 0.0027 & 2580.3 & 49.9 & 2664.3 & 34.9 & 2729.5 & 27.2 & 3.2 & 5.5 & 2729.5 & 27.2 \\
\hline G39 & 22.9 & 64.3 & 1.07 & 0.2834 & 0.0036 & 4.1095 & 0.0862 & 0.1052 & 0.0022 & 1608.4 & 36.2 & 1656.2 & 39.8 & 1718.3 & 34.5 & 2.9 & 6.4 & 1718.3 & 34.5 \\
\hline G40 & 159.8 & 304.9 & 0.43 & 0.4823 & 0.0056 & 12.8045 & 0.1928 & 0.1926 & 0.0028 & 2537.4 & 48.9 & 2665.4 & 34.8 & 2764.7 & 27.2 & 4.8 & 8.2 & 2764.7 & 27.2 \\
\hline G41 & 78.0 & 218.2 & 0.50 & 0.3250 & 0.0038 & 5.1918 & 0.0847 & 0.1159 & 0.0018 & 1814.1 & 37.4 & 1851.3 & 33.5 & 1894.1 & 26.9 & 2.0 & 4.2 & 1894.1 & 26.9 \\
\hline G42 & 139.0 & 242.0 & 0.28 & 0.5249 & 0.0062 & 14.0342 & 0.2182 & 0.1940 & 0.0029 & 2719.8 & 52.3 & 2752.0 & 35.9 & 2776.5 & 28.2 & 1.2 & 2.0 & 2776.5 & 28.2 \\
\hline G43 & 144.1 & 286.7 & 0.56 & 0.4531 & 0.0053 & 11.9810 & 0.1856 & 0.1919 & 0.0028 & 2408.9 & 47.3 & 2602.9 & 35.4 & 2758.3 & 28.1 & 7.5 & 12.7 & 2758.3 & 28.1 \\
\hline G44 & 115.4 & 261.6 & 0.12 & 0.4420 & 0.0052 & 11.1959 & 0.1768 & 0.1838 & 0.0028 & 2359.7 & 46.8 & 2539.6 & 35.8 & 2687.3 & 28.5 & 7.1 & 12.2 & 2687.3 & 28.5 \\
\hline G45 & 19.7 & 47.6 & 0.45 & 0.3762 & 0.0050 & 6.7594 & 0.1477 & 0.1304 & 0.0028 & 2058.3 & 46.7 & 2080.4 & 45.1 & 2103.2 & 38.7 & 1.1 & 2.1 & 2103.2 & 38.7 \\
\hline G46 & 47.6 & 110.5 & 0.96 & 0.3467 & 0.0043 & 5.5366 & 0.1038 & 0.1159 & 0.0021 & 1919.0 & 40.9 & 1906.3 & 38.1 & 1893.3 & 31.5 & -0.7 & -1.4 & 1893.3 & 31.5 \\
\hline G47 & 90.5 & 235.0 & 0.59 & 0.3406 & 0.0041 & 5.5027 & 0.0939 & 0.1172 & 0.0019 & 1889.3 & 39.1 & 1901.0 & 35.1 & 1914.6 & 28.5 & 0.6 & 1.3 & 1914.6 & 28.5 \\
\hline G48 & 30.3 & 76.9 & 0.61 & 0.3452 & 0.0045 & 5.4885 & 0.1189 & 0.1154 & 0.0025 & 1911.4 & 42.9 & 1898.8 & 43.3 & 1885.8 & 37.0 & -0.7 & -1.4 & 1885.8 & 37.0 \\
\hline G49 & 88.7 & 162.2 & 0.36 & 0.4991 & 0.0061 & 13.2681 & 0.2280 & 0.1929 & 0.0032 & 2609.8 & 52.6 & 2698.9 & 39.1 & 2767.1 & 31.7 & 3.3 & 5.7 & 2767.1 & 31.7 \\
\hline G50 & 255.6 & 482.5 & 0.30 & 0.4900 & 0.0057 & 12.5829 & 0.1956 & 0.1863 & 0.0028 & 2570.8 & 49.4 & 2648.9 & 35.5 & 2709.9 & 28.0 & 2.9 & 5.1 & 2709.9 & 28.0 \\
\hline G51 & 11.0 & 29.4 & 0.22 & 0.3615 & 0.0050 & 6.0796 & 0.1476 & 0.1220 & 0.0030 & 1989.2 & 46.9 & 1987.3 & 48.7 & 1986.1 & 42.4 & -0.1 & -0.2 & 1986.1 & 42.4 \\
\hline G52 & 95.2 & 155.3 & 0.54 & 0.5223 & 0.0062 & 13.4814 & 0.2220 & 0.1873 & 0.0030 & 2708.9 & 52.6 & 2714.0 & 37.4 & 2718.5 & 29.9 & 0.2 & 0.4 & 2718.5 & 29.9 \\
\hline G53 & 181.4 & 306.3 & 0.43 & 0.5231 & 0.0061 & 13.9677 & 0.2232 & 0.1937 & 0.0030 & 2712.3 & 52.0 & 2747.5 & 36.5 & 2774.2 & 29.0 & 1.3 & 2.2 & 2774.2 & 29.0 \\
\hline
\end{tabular}




\begin{tabular}{|c|c|c|c|c|c|c|c|c|c|c|c|c|c|c|c|c|c|c|c|}
\hline G54 & 126.7 & 215.4 & 0.61 & 0.4948 & 0.0059 & 11.6366 & 0.1961 & 0.1706 & 0.0028 & 2591.6 & 51.1 & 2575.6 & 37.7 & 2563.8 & 30.3 & -0.6 & -1.1 & 2563.8 & 30.3 \\
\hline G55 & 52.2 & 145.5 & 0.27 & 0.3436 & 0.0041 & 5.6066 & 0.1002 & 0.1184 & 0.0021 & 1903.9 & 39.7 & 1917.1 & 36.5 & 1932.2 & 30.1 & 0.7 & 1.5 & 1932.2 & 30.1 \\
\hline G56 & 7.4 & 14.0 & 0.22 & 0.5005 & 0.0084 & 13.5315 & 0.3900 & 0.1962 & 0.0058 & 2615.9 & 72.4 & 2717.5 & 64.1 & 2794.7 & 55.9 & 3.7 & 6.4 & 2794.7 & 55.9 \\
\hline G57 & 69.1 & 114.7 & 0.25 & 0.5466 & 0.0066 & 15.0746 & 0.2559 & 0.2001 & 0.0033 & 2811.1 & 54.9 & 2820.0 & 38.7 & 2827.0 & 31.1 & 0.3 & 0.6 & 2827.0 & 31.1 \\
\hline G58 & 80.0 & 124.5 & 0.87 & 0.5127 & 0.0062 & 13.3730 & 0.2286 & 0.1893 & 0.0031 & 2668.3 & 52.6 & 2706.4 & 38.6 & 2735.7 & 31.2 & 1.4 & 2.5 & 2735.7 & 31.2 \\
\hline G59 & 90.2 & 240.6 & 0.63 & 0.3280 & 0.0039 & 5.2967 & 0.0922 & 0.1172 & 0.0020 & 1828.5 & 38.0 & 1868.3 & 35.2 & 1913.7 & 29.0 & 2.1 & 4.4 & 1913.7 & 29.0 \\
\hline G60 & 232.3 & 430.8 & 0.26 & 0.5020 & 0.0059 & 13.1977 & 0.2170 & 0.1908 & 0.0030 & 2622.3 & 50.6 & 2693.9 & 37.1 & 2748.8 & 29.8 & 2.7 & 4.6 & 2748.8 & 29.8 \\
\hline G61 & 285.6 & 595.8 & 0.05 & 0.4759 & 0.0056 & 12.0270 & 0.1979 & 0.1834 & 0.0029 & 2509.3 & 48.8 & 2606.5 & 36.9 & 2683.6 & 29.7 & 3.7 & 6.5 & 2683.6 & 29.7 \\
\hline G62 & 67.6 & 106.3 & 0.54 & 0.5399 & 0.0066 & 14.8489 & 0.2600 & 0.1996 & 0.0034 & 2782.9 & 54.8 & 2805.6 & 39.6 & 2822.7 & 32.3 & 0.8 & 1.4 & 2822.7 & 32.3 \\
\hline G63 & 19.7 & 36.4 & 0.37 & 0.4938 & 0.0065 & 12.9942 & 0.2625 & 0.1909 & 0.0038 & 2587.1 & 55.8 & 2679.2 & 45.0 & 2750.2 & 37.8 & 3.4 & 5.9 & 2750.2 & 37.8 \\
\hline G64 & 84.0 & 162.2 & 0.49 & 0.4563 & 0.0056 & 10.2562 & 0.1856 & 0.1631 & 0.0029 & 2423.4 & 49.2 & 2458.2 & 39.5 & 2487.7 & 32.5 & 1.4 & 2.6 & 2487.7 & 32.5 \\
\hline G65 & 12.4 & 32.2 & 0.32 & 0.3591 & 0.0055 & 6.0101 & 0.1814 & 0.1214 & 0.0037 & 1977.9 & 51.9 & 1977.3 & 59.6 & 1977.5 & 53.3 & 0.0 & 0.0 & 1977.5 & 53.3 \\
\hline G66 & 356.9 & 693.8 & 0.05 & 0.5068 & 0.0060 & 13.6432 & 0.2297 & 0.1953 & 0.0032 & 2642.7 & 51.1 & 2725.3 & 37.8 & 2787.7 & 30.6 & 3.0 & 5.2 & 2787.7 & 30.6 \\
\hline G67 & 80.3 & 124.5 & 0.75 & 0.5270 & 0.0064 & 14.2360 & 0.2531 & 0.1960 & 0.0034 & 2728.8 & 53.9 & 2765.6 & 39.9 & 2793.1 & 32.7 & 1.3 & 2.3 & 2793.1 & 32.7 \\
\hline G68 & 122.3 & 237.8 & 0.26 & 0.4843 & 0.0058 & 12.7714 & 0.2228 & 0.1913 & 0.0032 & 2545.8 & 50.2 & 2662.9 & 38.8 & 2753.7 & 31.8 & 4.4 & 7.5 & 2753.7 & 31.8 \\
\hline G69 & 14.5 & 176.2 & 1.05 & 0.0659 & 0.0009 & 0.5061 & 0.0159 & 0.0557 & 0.0018 & 411.3 & 10.5 & 415.9 & 23.4 & 441.6 & 22.6 & 1.1 & 6.9 & 411.3 & 10.5 \\
\hline G70 & 32.1 & 50.4 & 0.99 & 0.4975 & 0.0064 & 12.7748 & 0.2533 & 0.1863 & 0.0036 & 2603.0 & 54.8 & 2663.2 & 43.8 & 2709.9 & 36.7 & 2.3 & 3.9 & 2709.9 & 36.7 \\
\hline G71 & 51.0 & 141.3 & 0.31 & 0.3426 & 0.0042 & 5.5187 & 0.1065 & 0.1169 & 0.0022 & 1899.0 & 40.2 & 1903.5 & 38.6 & 1909.0 & 32.4 & 0.2 & 0.5 & 1909.0 & 32.4 \\
\hline G72 & 61.4 & 114.7 & 0.43 & 0.4760 & 0.0058 & 11.1216 & 0.2073 & 0.1695 & 0.0031 & 2509.6 & 50.8 & 2533.4 & 40.7 & 2553.0 & 33.8 & 0.9 & 1.7 & 2553.0 & 33.8 \\
\hline G73 & 42.9 & 71.3 & 0.58 & 0.5144 & 0.0064 & 13.6049 & 0.2609 & 0.1919 & 0.0036 & 2675.2 & 54.7 & 2722.6 & 42.5 & 2758.5 & 35.5 & 1.7 & 3.0 & 2758.5 & 35.5 \\
\hline G74 & 36.1 & 111.9 & 0.28 & 0.3102 & 0.0039 & 4.6812 & 0.0961 & 0.1095 & 0.0022 & 1741.4 & 38.0 & 1763.9 & 39.7 & 1791.1 & 34.0 & 1.3 & 2.8 & 1791.1 & 34.0 \\
\hline G75 & 108.1 & 201.4 & 0.15 & 0.5103 & 0.0062 & 13.3803 & 0.2432 & 0.1902 & 0.0033 & 2657.8 & 52.5 & 2706.9 & 40.2 & 2744.2 & 33.3 & 1.8 & 3.1 & 2744.2 & 33.3 \\
\hline
\end{tabular}




\section{Osbornite}

Table DR3: Comparative geochemistry of all known occurrences of TiVN together with chemistry of TiNbN samples at sites 1 and 2 on Skye.

\begin{tabular}{|c|c|c|c|l|l|c|c|}
\hline & $\begin{array}{c}\text { Ti0.77V0.2 } \\
\text { 8Fe0.77N } \\
\text { Pure } \\
\text { Osbornite*, }\end{array}$ & $\begin{array}{l}\text { Osbornite } \\
\text { like phase } \\
\text { An Carnach }\end{array}$ & $\begin{array}{l}\text { Osbornite } \\
\text { Site 1 } \\
\text { Carnach } \\
\text { Site 1 }\end{array}$ & $\begin{array}{l}\text { Osbornite } \\
\text { An } \\
\text { Carnach } \\
\text { Site 1 }\end{array}$ & $\begin{array}{l}\text { Osbornite } \\
\text { Torrin } \\
\text { Road } \\
\text { Site 2 }\end{array}$ & $\begin{array}{l}\text { Osbornite } \\
\text { Torrin } \\
\text { Road } \\
\text { Site 2 }\end{array}$ & $\begin{array}{c}\text { Wild comet } \\
\text { Ti0.87V0.19Fe0 } \\
\text { 03N Chi M. et } \\
\text { al., 2009** }\end{array}$ \\
\hline $\mathrm{N}$ & 22.63 & 12.68 & 16.79 & 16.79 & 12.66 & 13.69 & 20.90 \\
\hline $\mathrm{S}$ & & 0.39 & 0.12 & 0.20 & 0.16 & 0.12 & \\
\hline $\mathrm{Ti}$ & 77.37 & 57.77 & 42.09 & 46.59 & 61.3 & 61.8 & 62.15 \\
\hline $\mathrm{V}$ & & 2.13 & 2.88 & 3.20 & 14.8 & 14.48 & 14.44 \\
\hline $\mathrm{Cr}$ & & 4.42 & 3.32 & 4.26 & 0.1 & 0.88 & \\
\hline $\mathrm{Fe}$ & & 2.89 & 3.01 & 2.98 & 2.34 & 2.22 & 2.51 \\
\hline $\mathrm{Nb}$ & & 9.73 & 11 & 14.16 & 0.9 & 0.65 & \\
\hline $\mathrm{C}$ & & 11.61 & & & & & \\
\hline & & & & & & & \\
\hline Total & 100 & 101.62 & 79.21 & 88.18 & 92.26 & 93.84 & 100.00 \\
\hline
\end{tabular}

\section{Osbornite References}

*Bannister, F. A., 1941, Osbornite meteoritic titanium nitride: Mineralogical Magazine, v. 26, p. 36-44.

${ }^{* *}$ Chi, M., Ishii, H. A., Simon, S. B., Bradley, J. P., Dai, Z., Joswiak, D. J., Browning, N. D. and Matrajt, G., 2009, The origin of refractory minerals in comet 81P/WILD 2: Geochimica et Cosmochimica Acta, v. 73(12), p. 71507161, doi:10.1016 /j.gca.2009.08.033. 


\section{Barringerite}

Table DR4: Comparative geochemistry of barringerite in selective meteorites, terrestrial Cu-Ni sulphides and the Skye impactoclastite sites.

\begin{tabular}{|c|l|c|c|c|c|c|c|}
\hline & $\begin{array}{l}\text { An } \\
\text { Carnach } \\
\text { Site 1 }\end{array}$ & $\begin{array}{l}\text { An } \\
\text { Carnach } \\
\text { Site 1 }\end{array}$ & $\begin{array}{l}\text { Torrin } \\
\text { Road } \\
\text { Site 2 }\end{array}$ & $\begin{array}{l}\text { Torrin } \\
\text { Road } \\
\text { Site 2 }\end{array}$ & $\begin{array}{c}\text { (Fe,Ni)2P } \\
\text { Buseck* } \\
1969 \\
\text { Meteorite } \\
\text { Ollague }\end{array}$ & $\begin{array}{l}\text { Brandstatter } \\
\text { et al** 1991 } \\
\text { Meteorite } \\
\text { Y-793274 }\end{array}$ \\
\hline $\mathrm{Si}$ & 0 & 0 & 0.36 & 0.5 & & & \\
\hline $\mathrm{P}$ & 15.36 & 0 & 14.4 & 12.71 & $\mathrm{P}$ & 21.8 & 22.8 \\
\hline $\mathrm{V}$ & 0 & 0 & 0.52 & 0.37 & $\mathrm{~V}$ & & \\
\hline $\mathrm{Cr}$ & 2.97 & 4.43 & 0.36 & 0.23 & $\mathrm{Cr}$ & & 0 \\
\hline $\mathrm{Mn}$ & 2.26 & 2.22 & 0.61 & 0.92 & $\mathrm{Mn}$ & & \\
\hline $\mathrm{Fe}$ & 79.33 & 82.22 & 81.78 & 80.31 & $\mathrm{Fe}$ & 44.3 & 75.1 \\
\hline $\mathrm{Ni}$ & 0.1 & 0.26 & 0.12 & 0.22 & $\mathrm{Ni}$ & 33.9 & 1.33 \\
\hline & & & & & $\mathrm{Co}$ & 0.25 & \\
\hline Total & 100.02 & 89.13 & 98.15 & 95.26 & Total & 100.25 & 0.21 \\
\hline & & & & & & & \\
\hline & & & & & & & 99.44 \\
\hline
\end{tabular}

\begin{tabular}{|c|c|c|}
\hline & $\begin{array}{l}\text { Chen et al } \\
1994^{* * *} \\
\text { Cu-Ni } \\
\text { Sulphide, } \\
\text { China }\end{array}$ & $\begin{array}{l}\text { Zolenski et al 2008**** } \\
\text { Kaidun Meteorite (FeCrP) } \\
\text { Andreyivanovite- barringerite } \\
\text { like phase }\end{array}$ \\
\hline$P$ & 20.21 & 22.32 \\
\hline $\mathrm{V}$ & 0.73 & 4 \\
\hline $\mathrm{Cr}$ & 0.73 & 21.99 \\
\hline \multicolumn{3}{|l|}{$\mathrm{Mn}$} \\
\hline $\mathrm{Fe}$ & 76.22 & 46.27 \\
\hline $\mathrm{Ni}$ & 2.85 & 2.54 \\
\hline $\mathrm{Ti}$ & & 2.79 \\
\hline $\mathrm{Co}$ & 0 & 0.08 \\
\hline Total & 100.01 & 99.99 \\
\hline
\end{tabular}

At both sites barringerite has very low $\mathrm{Ni}$ concentrations (0.1 - 0.21 wt. \%) but variable $\mathrm{Fe}, \mathrm{P}, \mathrm{Cr}, \mathrm{Mn}$ and $\mathrm{V}$. Such low Ni content is very similar to barringerite from the Y-793274 lunar meteorite (Brandstatter et al., 1991). However, $\mathrm{Cr}$ contents of Skye barringerite are closer to those of andreyivanovite ( $\mathrm{FeCrP}$ ) from the Kaidun meteorite (Zolenski et al., 2008). 


\section{Barringerite References}

* Buseck. P. R., 1969, Phosphide from meteorites: Barringerite, a new iron-nickel mineral: Science v. 165, p. 169171

** Brandstätter, F., Koeberl, C. and Gerokuat, N., 1991, The discovery of iron barringerite in lunar meteorite Y793274: Geochimica et Cosmochimica Acta, v. 55, p. 1173-1174.

*** Chen, K., Jin, Z. and Peng, Z., 1984, The discovery of iron barringerite (Fe,P) in China: Mineral. Abstracts, v. 35, p. 1871.

**** Zolensky, M., Gounelle, M., Mikouchi, T., Ohsumi, K., Le, L., Hagiya, K. and Tachikawa, O., 2008,

Andreyivanovite: A second new phosphide from the Kaidun meteorite: American Mineralogist v. 93, p. 1295-1299, doi:10.2138/am.2008.2614. 


\section{Reidite}

Table DR5: Raman peaks shown all meteorite impact studies (B-G) that have incorporated Raman analysis. Includes raman peaks from high pressure experimental data (A).

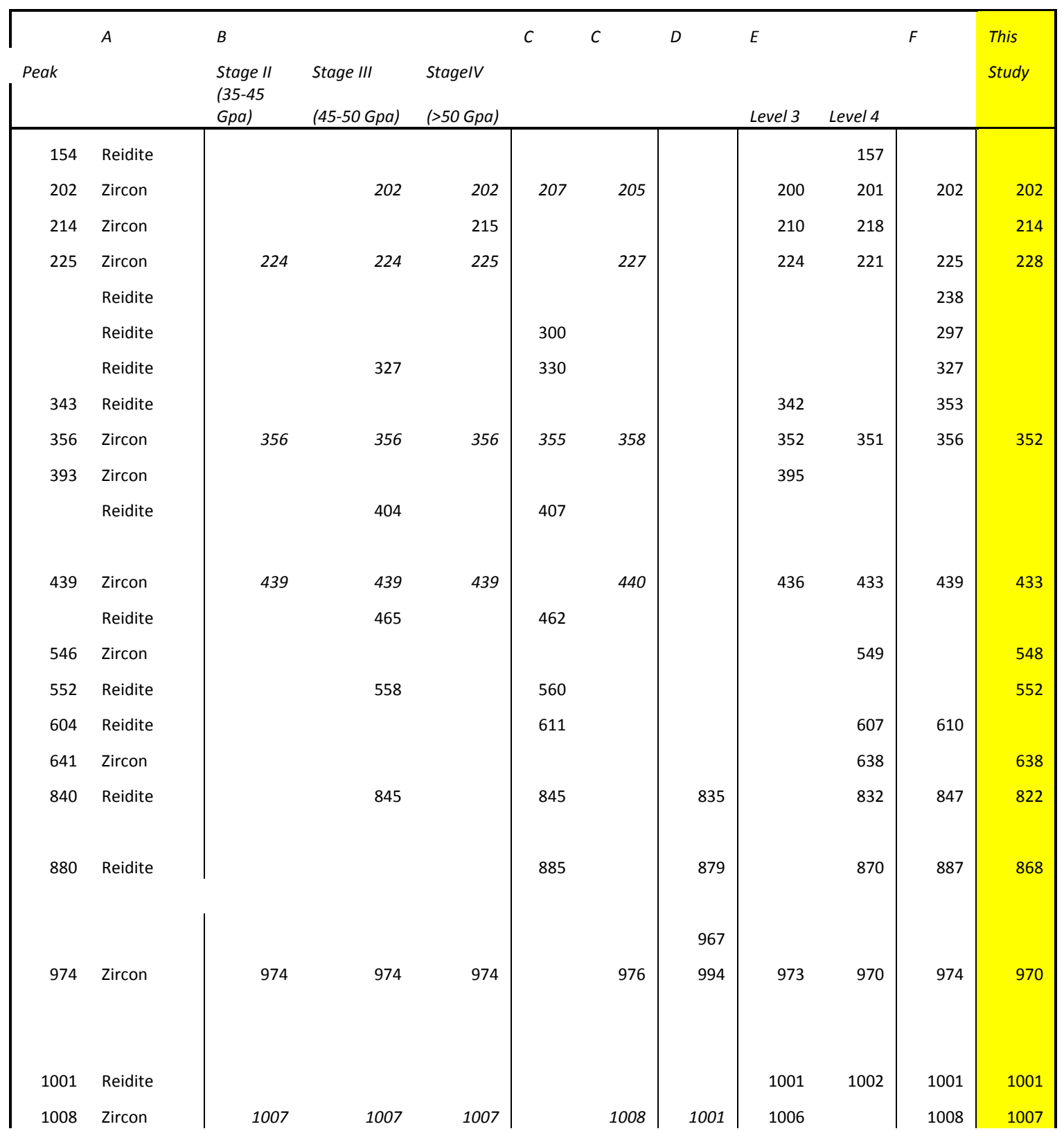




\section{Reidite References}

A. Knittle, E., and Williams, Q., 1993 High-pressure Raman spectroscopy of $\mathrm{ZrSiO}_{4}$ : Observation of the zircon to scheelite transition at $300 \mathrm{~K}$ : Am Mineral, v.78, p. 245-252.

B. Gucsik, A., 2006, $69^{\text {th }}$ Annual meteoritical identification of reidite from the Reis impact crater using microraman spectroscopy: a review.

C. Chen, M., Yin, F., Li, X., Xie, X., Xiao, W., and Tan, D., 2013, Natural occurrence of reidite in the Xiuyan crater of China: Meteorics and Planetary Science, v. 48, p. 796-805, doi:10.1111/maps.12106.

D. Malone, L., Boonsue, S., Spray, J. and Wittman, A., 2010, Zircon-reidite relations in breccias from Chesapeake Bay impact: 41st Lunar and Planetary Science Conference.

E. Singleton, A. C., Osinski, G. R. and Shieh, S. R., 2015, Microscope effects of shock metamorphism in zircon from the Haughton impact structure, Canada: GSA special paper, v. 518, p. 135-148, doi:10.1130/2015.2518(09).

F. Gucsik, A., 2007, Micro-Raman spectroscopy of reidite as an impact-induced high pressure polymorph of zircon: Experimental investigation and attempt to application: Acta Mineralogica-Petrographica, Szeged 2006-2007, v. 47, p. 17-24.

G. This study. 


\section{Alabandite}

Table DR6: Comparative geochemistry of alabandite in selective meteorites and the Skye impactoclastite sites.

\begin{tabular}{|c|l|l|l|l|l|l|}
\hline & $\begin{array}{l}\text { An Carnach } \\
\text { Site 1 }\end{array}$ & $\begin{array}{l}\text { An Carnach } \\
\text { Site 1 }\end{array}$ & $\begin{array}{l}\text { An Carnach } \\
\text { Site 1 }\end{array}$ & $\begin{array}{l}\text { Torrin Rd, } \\
\text { Site 2 }\end{array}$ & $\begin{array}{l}\text { Torrin Rd, } \\
\text { Site 2 }\end{array}$ & $\begin{array}{l}\text { Torrin Rd, } \\
\text { Site 2 }\end{array}$ \\
\hline $\mathrm{Mn}$ & 61.78 & 59.47 & 61.67 & 58.59 & 54.85 & 42.03 \\
\hline $\mathrm{Fe}$ & 1.52 & 0.00 & 0.00 & 0.00 & 0.00 & 31.36 \\
\hline $\mathrm{Mg}$ & 0.1 & 0.23 & 0.15 & 0.00 & 0.05 & 0.04 \\
\hline $\mathrm{Ca}$ & & 4.19 & & 9.67 & 7.87 & 0.00 \\
\hline $\mathrm{Cr}$ & & 1.19 & & & & \\
\hline $\mathrm{S}$ & 37.33 & 33.4 & 37.64 & 33.11 & 32.09 & 24.47 \\
\hline
\end{tabular}

\begin{tabular}{|l|l|l|l|l|l|l|l|l|}
\hline & $\begin{array}{l}\text { Torrin } \\
\text { Rd, } \\
\text { Site 2 }\end{array}$ & $\begin{array}{l}\text { Torrin } \\
\text { Rd, } \\
\text { Site 2 }\end{array}$ & $\begin{array}{l}\text { Torrin } \\
\text { Rd, } \\
\text { Site 2 }\end{array}$ & $\begin{array}{l}\text { Torrin } \\
\text { Rd, } \\
\text { Site 2 }\end{array}$ & $\begin{array}{l}\text { Torrin } \\
\text { Rd, } \\
\text { Site 2 }\end{array}$ & & $\begin{array}{l}\text { Ferroan } \\
\text { alabandite } \\
\text { Meteorite } \\
\text { Tupele (EL6) } \\
\text { *Dunlap et al., } \\
2013\end{array}$ & $\begin{array}{l}\text { Ferroan } \\
\text { alabandite } \\
\text { Meteorite. } \\
\text { EET 90102, } \\
\text { **Fogel, } \\
1997\end{array}$ \\
\hline $\mathrm{Mn}$ & 59.07 & 60.2 & 56.72 & 50.87 & 52.71 & $\mathrm{Mn}$ & 46.00 & 15.59 \\
\hline $\mathrm{Fe}$ & 7.25 & 7.14 & 12.14 & 14.02 & 13.58 & $\mathrm{Fe}$ & 14.72 & 41.86 \\
\hline $\mathrm{Mg}$ & 0.1 & 0.1 & 0.02 & 0 & 0.04 & $\mathrm{Mg}$ & 1.28 & 1.28 \\
\hline $\mathrm{S}$ & 32.88 & 33.65 & 32.04 & 30.19 & 30.77 & $\mathrm{~S}$ & 37.39 & 36.89 \\
\hline & & & & & & $\mathrm{Ca}$ & 0.19 & 2.24 \\
\hline & & & & & & $\mathrm{Cr}$ & 0.42 & 2.11 \\
\hline & & & & & & & & \\
\hline Total \% & 99.3 & 101.09 & 100.92 & 95.08 & 97.1 & Total & 100.00 & 99.97 \\
\hline & & & & & & & & \\
\hline
\end{tabular}

\section{Alabandite References}

* Dunlap, D. R., Pewit, M. L.,; McSween, H. Y., Taylor, L. A. and Doherty, R., 2013, Tupelo: A new El6 Enstatite Chondrite: 44th Lunar and Planetary Science Conference, abstract 2088.

*Fogel, R. A., 1997, On the significance of diopside and oldhamite in enstatite chondrites and aubrites: Meteoritics and Planetary Science, v. 32, p. 577-591. 
Table DR7: Site 2 (Chambered Cairn- lower unit) zircon ages

\begin{tabular}{|rccc|}
\hline Zircon & $\begin{array}{l}\text { 207Pb/206Pb } \\
\text { age }\end{array}$ & \pm 2 sigma & $\begin{array}{c}\text { Discordance } \\
\text { ( }\end{array}$ \\
& & & - \\
1 & 1819.9 & 27.8 & 1.8 \\
2 & 1737.9 & 3.4 & 0.2 \\
3 & 1340.4 & 3.7 & -1.5 \\
4 & 1111.8 & 13.8 & -4.3 \\
5 & 1489.8 & 18.2 & -1.9 \\
6 & 1492.4 & 12.3 & -0.8 \\
7 & 1633.2 & 15.1 & -1.9 \\
8 & 3227.1 & 3.1 & -3.7 \\
9 & 3219.4 & 2.5 & -1.1 \\
10 & 1765.9 & 6.0 & 0.4 \\
11 & 2826.0 & 6.0 & 0.4 \\
12 & 2813.6 & 3.1 & 0.8 \\
13 & 1179.3 & 9.5 & -0.9 \\
14 & 1720.1 & 8.3 & 0.9 \\
15 & 1728.7 & 6.2 & -3.0 \\
16 & 1719.7 & 6.2 & 1.1 \\
\hline
\end{tabular}




\section{Native Iron}

Table DR8: Chemistry of native iron within sites 1 and 2 on Skye.

\begin{tabular}{|c|c|c|c|l|l|l|}
\hline & $\begin{array}{c}\text { Native } \\
\text { metal An } \\
\text { Carnach } \\
\text { Site 1 }\end{array}$ & $\begin{array}{c}\text { Native } \\
\text { metal } \\
\text { Torrin Rd, } \\
\text { Site 2 }\end{array}$ & $\begin{array}{l}\text { Native } \\
\text { metal } \\
\text { Torrin Rd, } \\
\text { Site 2 }\end{array}$ & $\begin{array}{l}\text { Native } \\
\text { metal } \\
\text { Torrin Rd, } \\
\text { Site 2 }\end{array}$ & Minimum & Maximum \\
\hline $\mathrm{Si}$ & 1.55 & 0.8 & 1.45 & 2.31 & 0.8 & 2.31 \\
\hline $\mathrm{Cr}$ & 0.88 & 0.27 & 1.07 & 0.29 & 0.27 & 1.07 \\
\hline $\mathrm{Mn}$ & 1.23 & 0.1 & 1.37 & 0.66 & 0.1 & 1.37 \\
\hline $\mathrm{Fe}$ & 92.06 & 93.09 & 92.24 & 92.09 & 92.06 & 93.09 \\
\hline $\mathrm{Ni}$ & 0.14 & 0.12 & 0.11 & 0.49 & 0.11 & 0.49 \\
\hline $\mathrm{Cu}$ & 2.56 & 3.66 & 2.42 & 2.84 & 2.42 & 3.66 \\
\hline $\mathrm{C}$ & 1.12 & 2.18 & & & & \\
\hline Total \% & 100.54 & 100.22 & 98.66 & 98.68 & 95.76 & 101.99 \\
\hline
\end{tabular}

